\title{
Inverted Shifted Gompertz Distribution with Theory and Applications
}

\author{
Arun Kumar Chaudhary ${ }^{1}$, Laxmi Prasad Sapkota ${ }^{2}$, Vijay Kumar ${ }^{3}$ \\ ${ }^{1}$ Deparment of Management Science (Statistics), Nepal Commerce Campus, T.U., Kathmandu, Nepal. Email: akchaudhary1@yahoo.com, \\ ${ }^{2}$ Department of Statistics, Tribhuwan Multiple Campus, T.U., Tansen, Nepal. Email: laxmisapkota75@gmail.com \\ ${ }^{3}$ Department of Mathematics and Statistics, DDU Gorakhpur University, Gorakhpur, India. Email: vijay.mathstat@ddugu.acc.in
}

\begin{abstract}
:
In this work, a novel distribution called an inverted shifted Gompertz distribution by the inverse transformation method is introduced and studied. Some relevant characteristics of inverted shifted Gompertz distribution are mathematically and statistically discussed. To estimate parameters of an inverted shifted Gompertz distribution, some well-known estimation methods, namely LSE, MLE and CVME are used. $R$ software is applied for computational purposes. The applicability of the proposed model has been illustrated considering a real set of data and investigated the goodness-of-fit attained by the inverted shifted Gompertz model through various test statistics and graphical methods. We found that the proposed novel distribution is more flexible and delivered a better fit in contrast with some other selected lifespan models.
\end{abstract}

Keywords: CVME, Gompertz distribution, Inverted shifted Gompertz distribution, LSE, MLE.

\section{Introduction}

Gompertz (1824) first developed the conventional probability distribution called the Gompertz distribution which studies function of survival based on mortality laws and plays vital part in demonstrating human mortality and analyzing actuarial tables. Consider a random variable be denoted by $\mathrm{X}$ with parameters $\beta$ and $\lambda$ tracking the Gompertz model if its CDF can be expressed as

$$
F(x ; \lambda, \beta)=1-\exp \left\{\frac{\lambda}{\beta}(1-\exp (\beta x))\right\} ; x>0,(\beta, \lambda)>0
$$

It was used as a model for growth and also used to fit the tumor's growth. A large collection of data in life tables was condensed into a single function by the Gompertz function. It is based on the premise that as a person ages, the mortality rate decreases exponentially. For the number of individuals living as a function of age at a given age, the corresponding Gompertz function is. The Gompertz distribution, using real data, was used by (Ahuja \&Nash, 1967). The Gompertz-sinh family was introduced by (Cooray\&Ananda, 2010) and it was used to evaluate survival data with a strongly negative skewed.Some characteristics of the exponentiated distribution of Gompertz were demonstrated mathematically and statistically by (AbuZinadah\&Aloufi, 2014).El-Gohary et al.(2013) implemented a distribution termed generalized Gompertz distribution. Depending on the value of shape parameter, this model may havedecreasing or constant or increasing the bathtub curve failure rate. The PDF of the distribution of generalized Gompertz is stated by 


$$
g(t)=\beta \theta e^{\alpha t} \exp \left\{-\frac{\beta}{\alpha}\left(e^{\alpha t}-1\right)\right\}\left[1-\exp \left\{-\frac{\beta}{\alpha}\left(e^{\alpha t}-1\right)\right\}\right]^{\theta-1} ; \alpha \geq 0, \beta, \theta>0, t \geq 0
$$

Joshi \& Kumar (2020) has presented Logistic Gompertz distribution using Logistic-G family of distribution. A three-parameter power Gompertz distribution has been implemented by (Ieren et al., 2019) using a power transformation method. This study is inspired by the implementation of a new survival model that has a bathtub-shaped hazard function. Joshi \& Kumar (2020) has presented a new model using Gompertz distribution called Lindley Gompertz distribution and its CDF with parameters $(\alpha, \lambda, \theta)>0$ can be stated as

$$
G(x)=\left(1-\exp \left\{\frac{\lambda}{\alpha}[1-\exp (\alpha x)]\right\}\right)^{\theta}\left[1-\left(\frac{\theta}{1+\theta}\right) \log \left(1-\exp \left\{\frac{\lambda}{\alpha}[1-\exp (\alpha x)]\right\}\right)\right] ; x>0
$$

Based on the shape parameters' value, the hazard rate function of this distribution can show curve shape variations, such as an increasing or constant or decreasing or inverted bathtub curve. In this work, we have taken shifted Gompertz distribution as a base distribution. Bemmaor (1992) introduced the shifted Gompertz distribution whose CDF and PDF is

$$
\begin{aligned}
& F(t ; \alpha, \beta)=\left[1-\mathrm{e}^{-\beta t}\right] \exp \left\{-\alpha \mathrm{e}^{-\beta t}\right\} ; t \geq 0,(\alpha, \beta)>0 \\
& f(t ; \alpha, \beta)=\beta \exp (-\beta x+\alpha \exp (-\beta t)\{1+\alpha(1-\exp (-\beta t)\} ; t \geq 0,(\alpha \beta)>0
\end{aligned}
$$

Chaudhary \& Kumar (2020) introduced Gompertz Extension Distribution to measure its Bayesian estimation and prediction using the MCMC method.

To achieve a better fit, the key purpose of this work is to present a more flexible model for life-time data sets. The different sections of this paper have been arranged as follows; We define the inverted shifted Gompertz distribution along with some distributional characteristics. For the calculation of the parameter of the proposed distribution parameter, MLE, LSE and CVME methods are used and these are presented. Using a real dataset,we have conducted the goodness-of-fit test for determining the potentiality of the new distribution. In the last section, there are some concluding remarks.

\section{The Inverted Shifted Gompertz Distribution}

We have generated a novel distribution by employing an inverse transformation approach using shifted Gompertz distribution as parent distribution which we called inverse shifted Gompertz (ISGZ) distribution. Suppose $\mathrm{X}$ as random variable which follows inverse shifted Gompertz (ISGZ) distribution having $\alpha$ and $\theta$ as parameters, then its $\mathrm{CDF}$ is as follows:

$$
\begin{aligned}
& F(x)=1-\left(1-e^{-\theta / x}\right) \exp \left(-\alpha e^{-\theta / x}\right) ;(\alpha, \theta)>0, x>0 \\
& f(x)=\theta x^{-2}\left\{1+\alpha\left(1-e^{-\theta / x}\right)\right\} \exp \left(-\frac{\theta}{x}-\alpha e^{-\theta / x}\right) ; x>0,(\alpha, \theta)>0
\end{aligned}
$$

Survival /Reliability function of ISGZ model is

$$
S(x)=1-F(x)=\left(1-e^{-\theta / x}\right) \exp \{-\alpha \exp (-\theta / x)\} ; x>0,(\alpha, \theta)>0
$$

\section{Hazard rate function(HRF) of ISGZ distribution}

$$
h(x)=\frac{f(x)}{S(x)}=\frac{\theta x^{-2}\left\{1+\alpha\left(1-e^{-\theta / x}\right)\right\} \exp \left(-\frac{\theta}{x}-\alpha e^{-\theta / x}\right)}{\left(1-e^{-\theta / x}\right) \exp \left(-\alpha e^{-\theta / x}\right)} ;(\alpha, \theta)>0, x>0
$$

Fig. 1 shows PDF curve and HRF curve of the ISGZ distribution.. It has been found that there may be a different range of shapes for the PDF of ISGZ distribution. Due to its various shapes, such as increasing, increasing-decreasing, reverse bathtub for different values of parameter, the hazard rate function (HRF) for the ISGZ distribution is also versatile. 

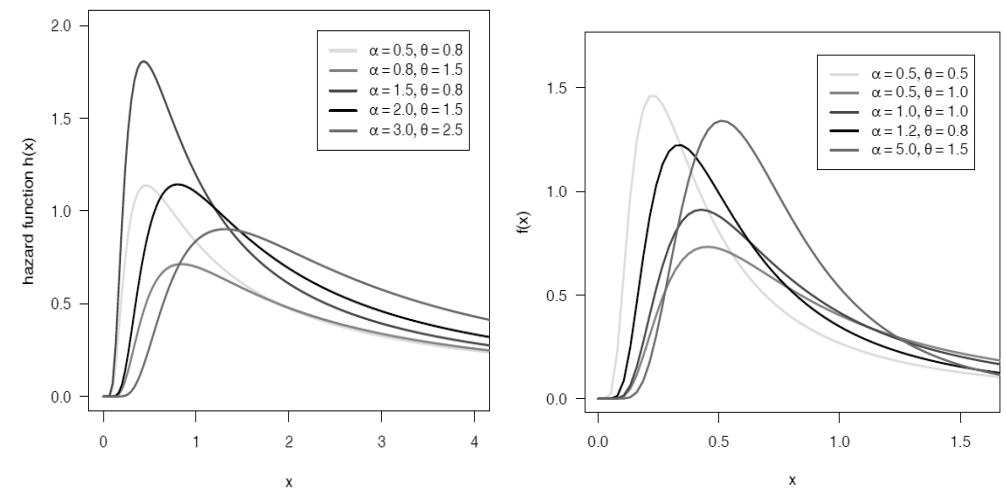

Fig. 1. PDF graph (right panel) and HRF graph (left panel) for various values of $\alpha$ and $\theta$.

\section{Quantile function of ISGZ distribution}

$$
\alpha e^{-\theta / x}-\ln (1-\exp (-\theta / x))+\ln (1-p)=0 ; 0<p<1
$$

For the creation of the random numbers of the ISGZ distribution, we assume the simulation of random variable $X$ values with the $\mathrm{CDF}(2.1)$. In $(0,1)$, let $\mathrm{B}$ denote a uniform random variable, then the simulated values of $X$ can be calculated as,

$$
\alpha \exp (-\theta / x)-\ln (1-\exp (-\theta / x))+\ln (1-b)=0 ; 0<b<1
$$

\section{Skewness and Kurtosis:}

The coefficient of skewness of ISGZ distribution can be obtained as

$$
S_{k}(B)=\frac{Q(3 / 4)+Q(1 / 4)-2 Q(1 / 2)}{Q(3 / 4)-Q(1 / 4)} \text { and }
$$

The coefficient of kurtosis given by (Moors, 1988) is

$$
K_{u}(M)=\frac{Q(0.875)-Q(0.625)+Q(0.375)-Q(0.125)}{Q(0.75)-Q(0.25)}
$$

\section{Methods of parameter estimation}

Estimation of ISGZ distribution's parameters using MLE method, LSE method and CVME method is done which is explained below.

\section{Maximum Likelihood Estimation (MLE) method}

Let with sample size ' $\mathrm{n}$ ' a random sample $\underline{x}=\left(x_{1}, \ldots, x_{n}\right)$ be drawn from $\operatorname{ISGZ}(\alpha, \theta)$ then the log-likelihood function can be expressed as

$$
\ell=n \ln \theta-2 \sum_{i=1}^{n} \ln x_{i}+\sum_{i=1}^{n} \ln \left\{1+\alpha\left(1-\exp \left(-\theta / x_{i}\right)\right)\right\}-\theta \sum_{i=1}^{n} \frac{1}{x_{i}}-\alpha \sum_{i=1}^{n} \exp \left(-\theta / x_{i}\right)
$$

By differentiating (3.1.1) w.r.t. unknown parameters $\alpha$ and $\theta$ we get

$$
\begin{aligned}
& \frac{\partial l}{\partial \alpha}=\sum_{i=1}^{n} \frac{1-\exp \left(-\theta / x_{i}\right)}{1+\alpha\left(1-\exp \left(-\theta / x_{i}\right)\right)}-\sum_{i=1}^{n} \mathrm{e}^{-\theta / x_{i}} \\
& \frac{\partial l}{\partial \theta}=\frac{n}{\theta}+\alpha \sum_{i=1}^{n} \frac{1}{x_{i}} \frac{1-\exp \left(-\theta / x_{i}\right)}{1+\alpha\left(1-\exp \left(-\theta / x_{i}\right)\right)}-\sum_{i=1}^{n} \frac{1}{x_{i}}+\alpha \sum_{i=1}^{n} \frac{1}{x_{i}} \exp \left(-\theta / x_{i}\right)
\end{aligned}
$$

Solving these non-linear equations for $(\alpha, \theta)$ by equating them to zero we will obtain the ML estimators of the ISGZ distribution. Computer software like R, Matlab, Mathematica, 
etc. can be used to solve them manually. Let $\underline{\omega}=(\alpha, \theta)$ denote the parameter vector and $\underline{\hat{\omega}}=(\hat{\alpha}, \hat{\theta})$ denote the corresponding MLE of $\underline{\omega}$ then $(\underline{\hat{\omega}}-\underline{\omega}) \rightarrow N_{2}\left[0,(D(\underline{\omega}))^{-1}\right]$ follows the normal distribution, where $D(\underline{\omega})$ represents the Fisher's information matrix found by,

$$
D=\left[\begin{array}{ll}
D_{11} & D_{12} \\
D_{21} & D_{22}
\end{array}\right]
$$

where,

$$
\begin{aligned}
& D_{11}=\frac{\partial^{2} l}{\partial \alpha^{2}}, D_{12}=\frac{\partial^{2} l}{\partial \alpha \partial \theta} \\
& D_{21}=\frac{\partial^{2} l}{\partial \theta \partial \alpha}, D_{22}=\frac{\partial^{2} l}{\partial \theta^{2}}
\end{aligned}
$$

Practically $\underline{\omega}$ unknown hence it is also unusable for the MLE to have an asymptotic variance $(D(\underline{\omega}))^{-1}$ . Placing estimated parameters value, asymptotic variance is approximated. Maximization of the likelihood with the help of the Newton-Raphson algorithm, generates the observed information matrix and the var-cov matrix is

$$
(D(\underline{\omega}))^{-1}=\left[\begin{array}{cc}
V(\hat{\alpha}) & C O V(\hat{\alpha}, \hat{\theta}) \\
\operatorname{COV}(\hat{\alpha}, \hat{\theta}) & V(\hat{\theta})
\end{array}\right]
$$

The MLEs' asymptotic normality can be constructed from approximately $100(1-\alpha)$ percent confidence intervals for $\alpha$ and $\theta$, as,

$$
\hat{\alpha} \pm Z_{\alpha / 2} S E(\hat{\alpha}) \text { and } \hat{\theta} \pm Z_{\alpha / 2} S E(\hat{\theta})
$$

\section{Least-Square Estimation (LSE)}

Another well-known method of estimation we have employed is a least-square estimation to estimate $\alpha$ and $\theta$ of ISGZ distribution, which can be found by minimizing

$$
C(X ; \alpha, \beta)=\sum_{i=1}^{n}\left[F\left(X_{i}\right)-\frac{i}{n+1}\right]^{2}
$$

w.r.t. $\alpha$ and $\theta$.

Consider $F\left(X_{i}\right)$ refers the $\mathrm{CDF}$ of the ordered random variables $\mathrm{X}_{(1)}<\mathrm{X}_{(2)}<\ldots<\mathrm{X}_{(\mathrm{n})}$ where with sample size $\mathrm{n}$, a random sample $\left\{X_{1}, X_{2}, \ldots, X_{n}\right\}$ is taken from $\mathrm{F}($.$) . The least-square estimators \hat{\alpha}$ and $\hat{\theta}$, can be found with minimization of

$$
C(X ; \alpha, \theta)=\sum_{i=1}^{n}\left[1-\left(1-\mathrm{e}^{-\theta / x_{i}}\right) \exp \left(-\alpha \exp \left(-\theta / x_{i}\right)\right)-\frac{i}{n+1}\right]^{2}
$$

with regard to $\alpha$ and $\theta$.

Differentiating (3.2.2) with respect to $\alpha$ and $\theta$ we get,

$$
\begin{aligned}
& \frac{\partial C}{\partial \alpha}=2 \sum_{i=1}^{n} e^{-\theta / x_{i}}\left[1-\left(1-\exp \left(-\theta / x_{i}\right)\right) \exp \left(-\alpha \exp \left(-\theta / x_{i}\right)\right)-\frac{i}{n+1}\right]\left(1-\exp \left(-\theta / x_{i}\right)\right) \exp \left(-\alpha e^{-\theta / x_{i}}\right) \\
& \frac{\partial C}{\partial \theta}=-2 \alpha \sum_{i=1}^{n}\left[1-\left(1-\exp \left(-\theta / x_{i}\right)\right) \exp \left(-\alpha e^{-\theta / x_{i}}\right)-\frac{i}{n+1}\right]\left(x_{i}^{-1} e^{-\theta / x_{i}} \exp \left(-\alpha e^{-\theta / x_{i}}\right)\left[1+\alpha\left(1-e^{-\theta / x_{i}}\right)\right]\right.
\end{aligned}
$$

Similarly, by minimizing $W(X ; \alpha, \theta)$ w.r.t. $\alpha$ and $\theta$, the weighted least square estimators can be acquired. 
$W(X ; \alpha, \theta)=\sum_{i=1}^{n} w_{i}\left[F\left(X_{(i)}\right)-\frac{i}{n+1}\right]$

The weights $w_{i}$ are $w_{i}=\frac{1}{\operatorname{Var}\left(X_{(i)}\right)}=\frac{(n+1)^{2}(n+2)}{i(n-i+1)}$

Hence, by minimizing $W(X ; \alpha, \theta)$ w.r.t. $\alpha$ and $\theta$ respectively, the weighted LSE of $\alpha$ and $\theta$ can be calculated.

$$
W(X ; \alpha, \theta)=\sum_{i=1}^{n} \frac{(n+1)^{2}(n+2)}{i(n-i+1)}\left[1-\left(1-e^{-\theta / x_{i}}\right) \exp \left(-\alpha e^{-\theta / x_{i}}\right)-\frac{i}{n+1}\right]^{2}
$$

w.r.t. $\alpha$ and $\theta$.

\section{Cramer-Von-Mises estimation (CVME)}

By minimizing the function, the CVMEs of $\alpha$ and $\theta$ are established.

$$
D(X ; \alpha, \theta)=\frac{1}{12 n}+\sum_{i=1}^{n}\left[F\left(x_{i: n} \mid \alpha, \theta\right)-\frac{2 i-1}{2 n}\right]^{2}=\frac{1}{12 n}+\sum_{i=1}^{n}\left[1-\left(1-\mathrm{e}^{-\theta / x_{i}}\right) \exp \left(-\alpha e^{-\theta / x_{i}}\right)-\frac{2 i-1}{2 n}\right]^{2}
$$

Differentiating (3.3.1) w.r.t. $\alpha$ and $\theta$, we get,

$$
\begin{aligned}
& \frac{\partial D}{\partial \alpha}=2 \sum_{i=1}^{n} e^{-\beta / x_{i}}\left[1-\left(1-e^{-\theta / x_{i}}\right) \exp \left(-\alpha e^{-\theta / x_{i}}\right)-\frac{2 i-1}{2 n}\right]\left(1-\mathrm{e}^{-\theta / x_{i}}\right) \exp \left(-\alpha \exp \left(-\theta / x_{i}\right)\right) \\
& \frac{\partial D}{\partial \theta}=-2 \alpha \sum_{i=1}^{n}\left[1-\left(1-\exp \left(-\theta / x_{i}\right)\right) \exp \left(-\alpha \exp \left(-\theta / x_{i}\right)\right)-\frac{2 i-1}{2 n}\right] x_{i}^{-1} e^{-\theta / x_{i}} \exp \left(-\alpha e^{-\theta / x_{i}}\right)\left[\alpha\left(1-e^{-\theta / x_{i}}\right)+1\right]
\end{aligned}
$$

simultaneously.

We can obtain the CVM estimators after solving non-linear expressions $\frac{\partial D}{\partial \alpha}=0$ and $\frac{\partial D}{\partial \theta}=0$

\section{Application To Real Dataset}

Here, we illustrated the suitability and applicability of the ISGZ distribution using a real data set employing by previous investigators. Here, we fit our model with the tensile strength data of 65 failure stress observations of single $50 \mathrm{~mm}$ long carbon fibers. The data were also applied by (Bader \& Priest, 1982) and discussed later in (Muhammad \& Liu, 2019). The data were as follows:

$1.339,1.434,1.549,1.574,1.589,1.613,1.746,1.753,1.764,1.807,1.812,1.84,1.852,1.852,1.862$, $1.864,1.931,1.952,1.974,2.019,2.051,2.055,2.058,2.088,2.125,2.162,2.171,2.172,2.18,2.194,2.211$, $2.27,2.272,2.28,2.299,2.308,2.335,2.349,2.356,2.386,2.39,2.41,2.43,2.431,2.458,2.471,2.497$, $2.514,2.558,2.577,2.593,2.601,2.604,2.62,2.633,2.67,2.682,2.699,2.705,2.735,2.785,3.02,3.042$, $3.116,3.174$

By utilizing R software (R Core Team, 2020) of the optim () function, we have calculated the MLEs of ISGZ distribution via maximizing the likelihood function (3.1.1) (Mailund, 2017). We have obtained the value of Log-Likelihood is $l=-34.8995$. We have shown the MLEs with their 95 percent confidence intervals for $\alpha$ and $\theta$ and standard errors (SE) in Table 1 .

Table 1

MLE and SE and $95 \%$ confidence interval for $\alpha$ and $\theta$ of ISGZ

\begin{tabular}{lccc}
\hline Parameter & MLE & SE & 95\% CI \\
\hline $\mathrm{A}$ & 215.8181 & 8.7756 & $(198.6179,233.0183)$ \\
$\Theta$ & 12.7678 & 0.3222 & $(12.1363,13.3993)$ \\
\hline
\end{tabular}

In Figure 2, the contour plot and fitted CDF with empirical distribution function (EDF) of ISGZ 

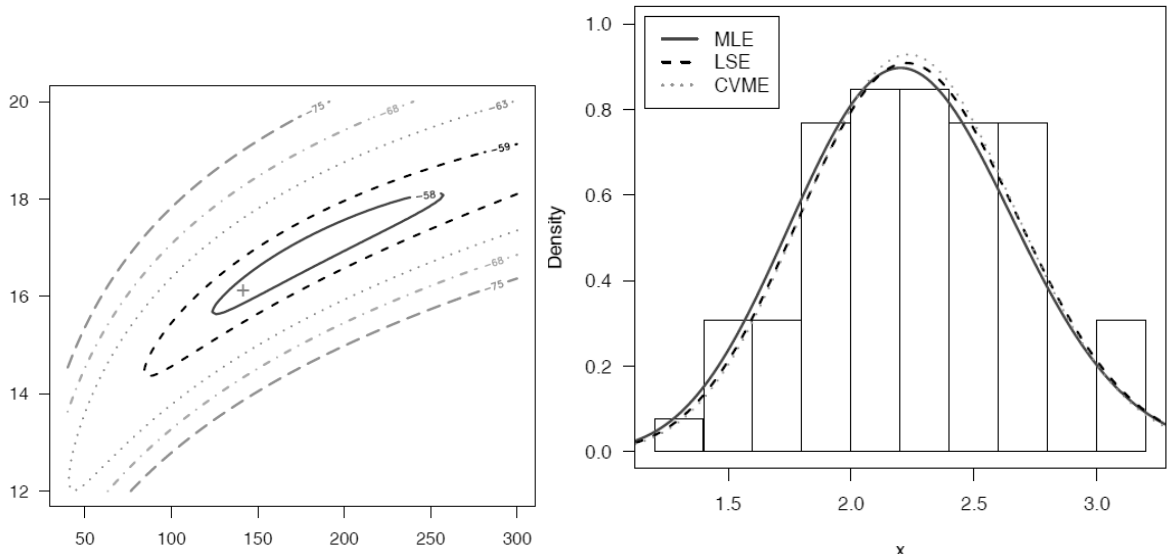

Figure 2. Contour plot (left panel) and the Histogram and the density function of fitted distributions (right panel) of estimation methods MLE, LSE and CVM of ISGZ distribution.

The plots of the log-likelihood function's profile for $\alpha$ and $\theta$ are displayed in Figure 3 and found that the ML estimates can be calculated uniquely.
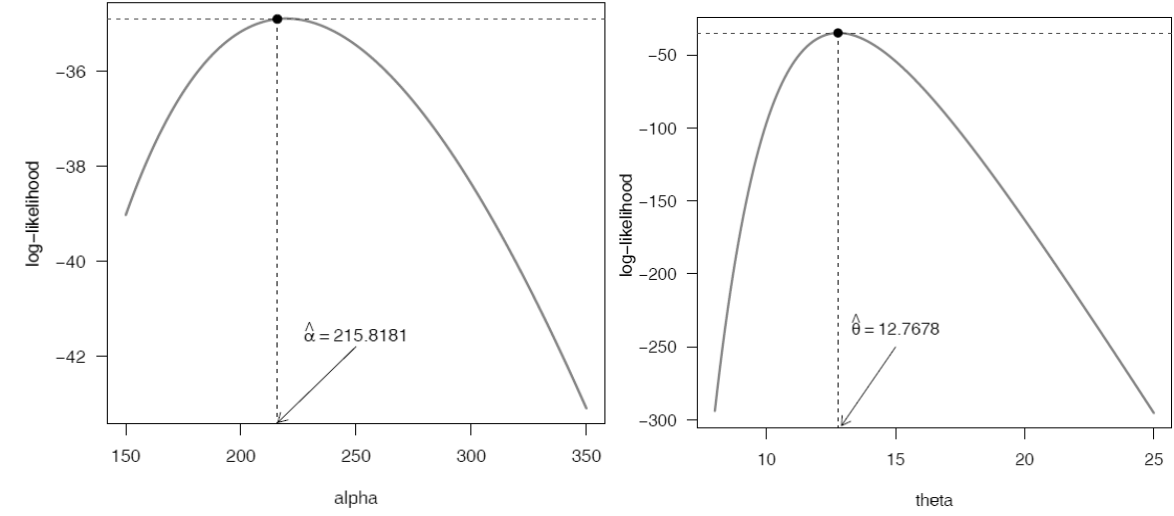

Figure 3. Profile log-likelihood function's graph for the parameters $\alpha$ and $\theta$.

In Fig 4, we have presented the graph of P-P plot and Q-Q plot and it is found that the ISGZ distribution fits the data very well.
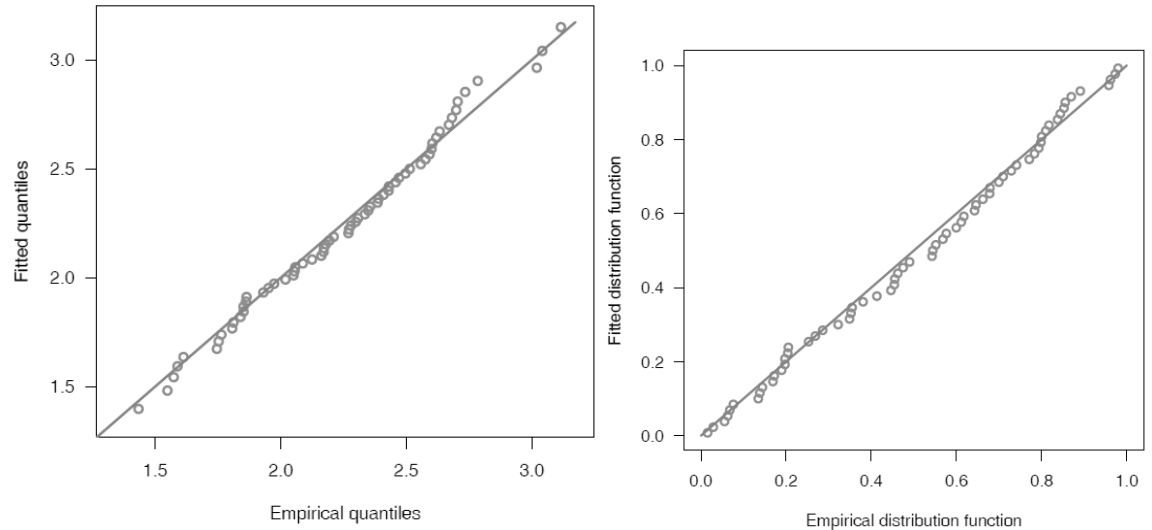

Figure 4. The plots of Q-Q (left panel) and P-P (right panel) of the ISGZ distribution.

Using MLE, LSE and CVE method we have displayed the estimated values of $\alpha$ and $\theta$ of ISGZ distribution and their corresponding negative log-likelihood, AIC and BIC criterion in Table 2. 
Table 2

Estimated parameters, log-likelihood, AIC and BIC

\begin{tabular}{lccccc}
\hline Method of Estimation & $\hat{\alpha}$ & $\hat{\theta}$ & -LL & AIC & BIC \\
\hline MLE & 215.8181 & 12.7678 & 34.8995 & 73.7989 & 78.1477 \\
LSE & 246.2792 & 13.1890 & 34.7441 & 73.4882 & 77.8370 \\
CVE & 282.0271 & 13.4975 & 34.6001 & 73.2003 & 77.5490 \\
\hline
\end{tabular}

The KS, W and $\mathrm{A}^{2}$ statistic with their corresponding p-value of MLE, LSE and CVE estimates we have presented in Table 3 .

Table 3

The KS, W and A2 statistic with a p-value

\begin{tabular}{lccc}
\hline Method of Estimation & KS(p-value $)$ & W(p-value $)$ & A2(p-value $)$ \\
\hline MLE & $0.0657(0.9416)$ & $0.0433(0.9171)$ & $0.2772(0.9541)$ \\
LSE & $0.0597(0.9745)$ & $0.0279(0.9833)$ & $0.2209(0.9836)$ \\
CVE & $0.0626(0.9608)$ & $0.0268(0.9860)$ & $0.2073(0.9883)$ \\
\hline
\end{tabular}

In this section, we have presented the applicability of Poisson shifted Gompertz distribution using a real dataset used by earlier researchers. To compare the potentiality of the proposed model, we have considered the following four distributions.

a) Gompertz distribution (GZ):

The PDF of Gompertz distribution (Murthy et al., 2003) having $\alpha$ and $\theta$ is

$$
f_{G Z}(x)=\theta e^{\alpha x} \exp \left\{\frac{\theta}{\alpha}\left(1-e^{\alpha x}\right)\right\} ; x \geq 0, \theta>0,-\infty<\alpha<\infty .
$$

b) Generalized Gompertz (GG) distribution

The PDF of GG distribution (El-Gohary et al., 2013) with parameters $\alpha, \lambda$ and $\theta$ is

$$
f_{G G Z}(x)=\theta \lambda e^{\alpha x} e^{-\frac{\lambda}{\alpha}\left(e^{\alpha x}-1\right)}\left[1-\exp \left(-\frac{\lambda}{\alpha}\left(e^{\alpha x}-1\right)\right)\right]^{\theta-1} ; \lambda, \theta>0, \alpha \geq 0, x \geq 0
$$

c) Shifted Gompertz (SG) distribution

The SG distribution (Bemmaor, 1992) 's PDF is

$$
f_{S G Z}(t ; \alpha, \beta)=\beta \exp \left(-\beta x+\alpha e^{-\beta t}\right)\left\{1+\alpha\left(1-e^{-\beta t}\right)\right\} ; t \geq 0,(\alpha \beta)>0
$$

d) Generalized Exponential Extension (GEE) distribution:

GEE's PDF developed by (Lemonte, 2013) with $\alpha, \beta$ and $\lambda$ which is as follows.

$$
f_{G E E}(x ; \alpha, \beta, \lambda)=\alpha \beta \lambda(1+\lambda x)^{\alpha-1} \exp \left\{1-(1+\lambda x)^{\alpha}\right\}\left[1-\exp \left\{1-(1+\lambda x)^{\alpha}\right\}\right]^{\beta-1} ; x \geq 0 .
$$

\section{e) Weibull Extension (WE) Model:}

The probability density function of Weibull extension (WE) distribution (Tang et al., 2003) with three parameters $(\alpha, \beta, \lambda)$ is

$$
f_{W E}(x ; \alpha, \beta, \lambda)=\lambda \beta\left(\frac{x}{\alpha}\right)^{\beta-1} \exp \left(\frac{x}{\alpha}\right)^{\beta} \exp \left\{-\lambda \alpha\left(\exp \left(\frac{x}{\alpha}\right)^{\beta}-1\right)\right\} ; x>0
$$


$\alpha>0, \beta>0$ and $\lambda>0$

For the evaluation of the applicability of the ISGZ distribution, we have illustrated the AIC, BIC, CAIC and HQIC which is shown in Table 4.

Table 4

Log-likelihood (LL), AIC, BIC, CAIC and HQIC

\begin{tabular}{lccccc}
\hline Model & -LL & AIC & BIC & CAIC & HQIC \\
\hline ISGZ & 34.8995 & 73.7989 & 78.1477 & 73.9925 & 75.5148 \\
GGZ & 34.5941 & 75.1883 & 81.7115 & 75.5817 & 77.7621 \\
GEE & 35.0445 & 76.0889 & 82.6121 & 76.4824 & 78.6627 \\
WE & 35.4743 & 76.9485 & 83.4717 & 77.3420 & 79.5223 \\
SGZ & 38.3586 & 80.7172 & 85.0660 & 80.9108 & 82.4331 \\
GZ & 38.9102 & 81.8205 & 86.1692 & 82.0140 & 83.5363 \\
\hline
\end{tabular}

We have presented the plot of goodness-of-fit of ISGZ distribution and some selected distributions are in Figure 5.
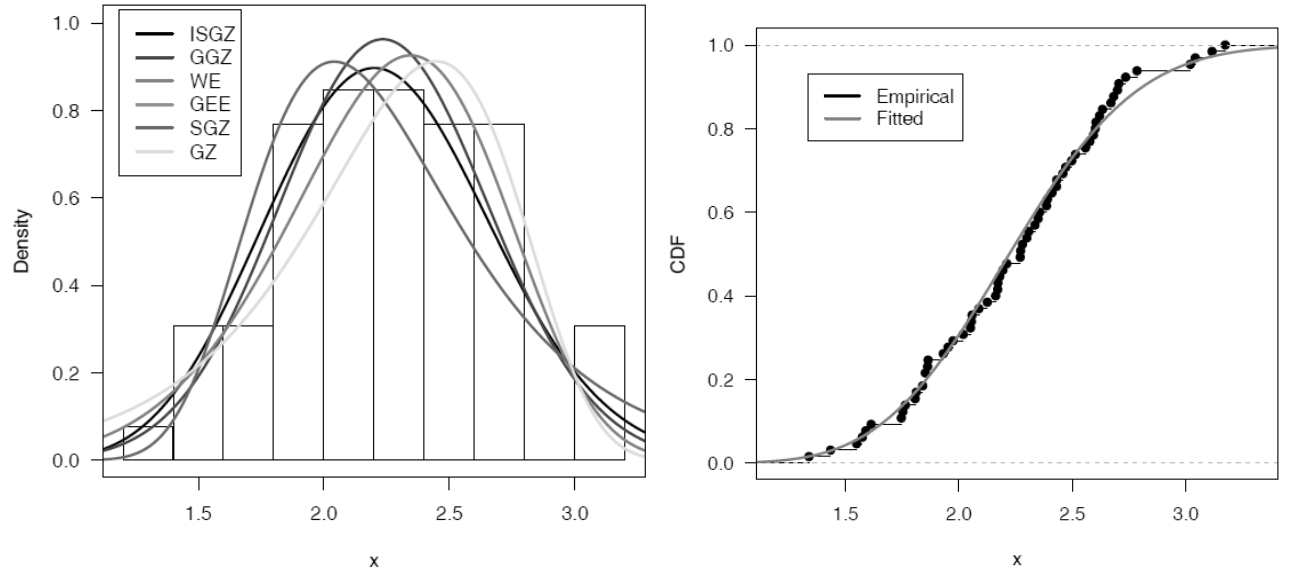

Figure 5. Fitted distributions's PDF and Histogram (left panel) and CDF of Empirical with fitted distribution (right panel)

We have also shown the value of Anderson-Darling (AD) Kolmogorov-Simnorov (KS), and CramerVon Mises (CVM) statistics in Table 5 for comparing the goodness-of-fit of the ISGZ distribution with former rival models. It is noted that the ISGZ distribution has the minimum value and higher p-value of the test statistics, so we infer that the ISGZ distribution gets quite a better fit from others taken for comparison.

Table 5

The goodness-of-fit statistics with their p-value

\begin{tabular}{lccc}
\hline Model & KS(p-value $)$ & $\mathrm{AD}(\mathrm{p}$-value $)$ & CVM(p-value $)$ \\
\hline ISGZ & $0.0657(0.9416)$ & $0.0433(0.9171)$ & $0.2772(0.9541)$ \\
GGZ & $0.0665(0.9359)$ & $0.0299(0.9772)$ & $0.2154(0.9856)$ \\
GEE & $0.0723(0.8865)$ & $0.0539(0.8541)$ & $0.3244(0.9185)$ \\
WE & $0.0558(0.9875)$ & $0.0264(0.9871)$ & $0.2799(0.9523)$ \\
SGZ & $0.0923(0.6369)$ & $0.1212(0.4915)$ & $0.8037(0.4779)$ \\
GZ & $0.0697(0.9107)$ & $0.0799(0.6939)$ & $0.7486(0.5191)$ \\
\hline
\end{tabular}




\section{Concluding Remarks}

We have developed a new probability model called inverted shifted Gompertz distribution. Some of the significant characteristics of the new model are presented, such as the derivation of explicit terms for its failure rate function, reliability function, quantile function, skewness, and kurtosis functions.. MLE, LSE, and CVME methods, are used for the parameter estimation and we found that the MLEs are reasonably good than LSE and CVMM methods. The curves of the PDF of the proposed distribution have shown that its shape is increasing-decreasing and right-skewed and flexible for modeling real-life data. Also, the graph of the failure function is monotonically increasing or constant or reverse $j$-shaped according to the parameters values. The applicability and suitability of the recommended novel model have been evaluated by considering a true dataset and the results exposed that the recommended distribution is much versaltile as compared to some other fitted distributions.

\section{References}

Abu-Zinadah, H. H., \&Aloufi, A. S. (2014). Some characterizations of the exponentiatedGompertz distribution. In International Mathematics Forum (Vol. 9, No. 30, pp. 1427-1439).

Ahuja, J.C. \& Nash, S.W. (1967). The generalized Gompertz-Verhulst family of distributions, Sankhya, Part A, 29, 141-156.

Bader, M. G., \& Priest, A. M. (1982). Statistical aspects of fiber and bundle strength in hybrid composites. Progress in science and engineering of composites, 1129-1136.

Chaudhary, A.K. \& Kumar, V. (2020). A Bayesian Estimation and Prediction of Gompertz Extension Distribution Using the MCMC Method. Nepal Journal of Science and Technology (NJST),19(1),142160.ISSN:1994-1412.

Cooray, K., \&Ananda, M. M. (2010). Analyzing survival data with highly negatively skewed distribution: The Gompertz-sinh family. Journal of Applied Statistics, 37(1), 1-11.

El-Gohary, A., Alshamrani, A., \& Al-Otaibi, A. N. (2013). The generalized Gompertz distribution. Applied Mathematical Modelling, 37(1-2), 13-24.

Gompertz, B. (1824). On the nature of the function expressive of the law of human mortality and on the new mode of determining the value of life contingencies, Phil. Trans. Royal Soc. A, 115, 513-580.

Ieren, T. G., Kromtit, F. M., Agbor, B. U., Eraikhuemen, I. B., \&Koleoso, P. O. (2019). A power Gompertz distribution: Model, properties and application to bladder cancer data. Asian Research Journal of Mathematics, 1-14.

Joshi, R. K. \& Kumar, V.(2020). Lindley Gompertz distribution with properties and application. International Journal of Statistics and Applied Mathematics, 5(6), 28-37.

Joshi, R. K. \& Kumar, V. (2020). The Logistic Gompertz Distribution with Properties and Applications. Bull. Math. \& Stat. Res.,8(4),81-94.

Kumar, V. and Ligges, U. (2011). reliaR: A package for some probability distributions, http://cran.r-project. org/web/packages/reliaR/index.html.

Lemonte, A. J. (2013). A new exponential-type distribution with constant, decreasing, increasing, upsidedown bathtub and bathtub-shaped failure rate function. Computational Statistics \& Data Analysis, 62, 149-170.

Mailund, T. (2017). Functional Programming in R: Advanced Statistical Programming for Data Science, Analysis and Finance. Apress, Aarhus N, Denmark ISBN-13 (pbk): 978-1-4842-2745-9 ISBN-13 (electronic): 978-1-4842-2746-6 DOI 10.1007/978-1-4842-2746-6

Moors, J. (1988). A quantile alternative for kurtosis. The Statistician, 37, 25-32.

Muhammad, M., \& Liu, L. (2019). A New Extension of the Generalized Half Logistic Distribution with Applications to Real Data. Entropy, 21(4), 339.

Murthy, D.N.P., Xie, M. and Jiang, R. (2003). Weibull Models, Wiley, New York.

R Core Team (2020). R: A language and environment for statistical computing. R Foundation for Statistical 
Issue 1 May/June 2020

Computing, Vienna, Austria. URL https://www.R-project.org/.

Tang, Y., Xie, M., \&Goh, T. N. (2003). Statistical analysis of a Weibull extension model. Communications in Statistics-Theory and Methods, 32(5), 913-928. 